\title{
A New Trend of Digital Healthcare in 3D Printed Medicines
}

\author{
Itimad Raheem Ali ${ }^{1}$, Jwan K. Alwan ${ }^{2}$, Dhulfiqar Saad Jaafar ${ }^{3}$, Hoshang Kolivand ${ }^{4}$ \\ ${ }^{1}$ MIS Department, College of Business Informatics, University of Information Technology and Communications, \\ Baghdad, Iraq. \\ ${ }^{2}$ Biomedical Informatics Collage, University of Information Technology and Communications, Baghdad, Iraq \\ ${ }^{3}$ Minisitry of Education, Baghdad, Iraq. \\ ${ }^{4}$ Department of Computer Science, Liverpool John Moores University, L3 3AF, Liverpool, UK \\ Correspondence: Itimad Raheem Ali, MIS Department, College of Business Informatics, University of \\ Information Technology and Communications, Baghdad, Iraq.
}

Received: May 25, 2020

doi:10.5539/cis.v13n4p12
Accepted: July 16, 2020

Online Published: September 30, 2020

URL: https://doi.org/10.5539/cis.v13n4p12

\begin{abstract}
Three-dimensional (3D) technique of restricting scrambling is changing the ways of drug design, labeling and production in the area of digital health. By combining digital and genetic techniques, Fused Deposition Modeling (FDM) can manufacture normalization systems. Consecutively, such a method can allow for speedy improvements in the healthcare systems, allowing the allocation of medicines based on patient's needs and requirements. So far, several 3D based medicinal goods have been marketed. These include the production of implants and several useful related products for use in medical applications. Nevertheless, regulatory obstacles remain with developing medicines. This article reviews the latest FDM technology in medical and pharmaceutical research, including a discussion of the potential challenges in the field. Emphasis has been paid on future developments needed for facilitating the FDM integration into dispensaries and clinics.
\end{abstract}

Keywords: drug delivery systems, patient-centric medications, personalized medicines fused filament fabrication, fused deposition modeling, material extrusion

\section{Introduction}

Three-dimensional (3D) technology is known to gain an increasing amount of popularity in several existing and upcoming manufacturing businesses. It is now considered as an innovative tooling method. This additional system changes the manner in which products are produced and designed and improves such processes. With 3D numerical modelling (Wink M., 2015), custom products can be produced with the assistance of automation techniques. Hence, this method is able to create a successful link between realism and ingenuity. Several 3D modelling techniques are available commercially (Ali. Itimad et al., 2019). Fused Deposition Modeling (FDM) is widely being used in several other industries as well, such as the footwear, jewelry, arts, dentistry, health care and automobiles as well as aviation. It is worth mentioning that FDM is a well-known technique, which is currently in use around the world, specifically in pharmaceuticals. Hence, FDM is deemed as an appropriate technique for use in digital health (Fina et al., 2012). Moreover, it is expected that adequate implementation of FDM in the pharmaceutical field can result in advancements in pharmaceutical formulation, design and production areas (Kyobula et al., 2017). Particularly, FDM can be implemented as a manufacturing technique in digital health for manufacturing as well as to distribute customized kits with improved dosages, sizes as well as shapes for patients remotely. These advantages offer the flexibility and independence in the treatment procedure, which may result in enhanced treatment and adherence to medication (Mohamed et al., 2015). This review provides an overview of FDM 3DM's revolutionary possibilities and opportunities in drug production as well as formulation. The review intends to highlight the exceptionally regulatory as well as technical challenges, which need to be taken into account prior to being developed for common applications.

\section{Extraction of Substances}

The precept of popular extrusion of substances is a thermal procedure; the essential precept is the selective elimination of magma via a gap to generate stable semi-strong filaments, which harden at a constructing plate for creating 3-dimensional gadgets. The utilized materials in this approach consist of 
thermoplastics, clay, candles, pastes along with gels. Intrinsically, extrusion is a huge time period that extends to different salient strategies, together with FDM and semi-metal extrusion (Hospodiuk et al., 2015). While FDM are used interchangeably, FDM handiest offers with thermoplastic substances. FDM generation, additionally called molten fuse fabrication (FFF), turned into a creation with the aid of Scott Cromb in 1988 when he started making a toy for his daughter with the use of an easy glue-gun. He changed the traditional glue stick with an aggregate of polyethene wax and candles and used it to construct sport layers separately. The concept became then elevated and a technique replica became advanced. In 1989, Cromb and his spouse were given a patented generation and co-based their personal agency, Stratus, to marketplace their merchandise. This generation changed into formulated as a logo call FDM (Bhaduri A., 2018).

In terms of the timeline of the modern-day improvements within the FDM manner, similar to another 3DM era, the FDM technique follows "the 3D's of 3DM". The procedure is: (a) Design: a 3D form is designed with the use of a computer-aided-design (CAD) program. The form is numerically split into several horizontal layers. Next, the form is applied into the software program in the shape of a stereo lithography (stl.) record, (b) increase: an appropriate drug loaded, thermoplastic filament is evolved after which it is applied in the printer, extruded as well as melted via a metallic outlet at selected temperatures. Usually, printers permit person-choice for the printing head temperature. The FDM printer may be utilized for printing extraordinary polymer blends as well as polymers; (c) dispensing: the filament which is extruded gets dropped at the levelled bottom of a printer, referred to as the construct platform or plate. Further, the print head actions in a raster sample, for creating a primary layer for the item. With final touches of every layer, the platform is decreased to permit sufficient area for a brand-new layer deposition. With the cooling down of the filament, the filament gets attached to the preceding layer (Wagner $\mathbf{J}$ et al., 2013). To achieve completeness of the item, a repetition of the technique takes place (Figure 1). The decision of the broadcast item depends on the extruded filament's thickness, which is commonly one hundred $\mu \mathrm{m}$ in an industrial printer. By ensuring that the conversion of the polymer takes place, FDM possesses the capability of creating complicated gadgets possessing specific traits, along with excessive mechanical power as well as thermal resistance (Kutz M., 2011).

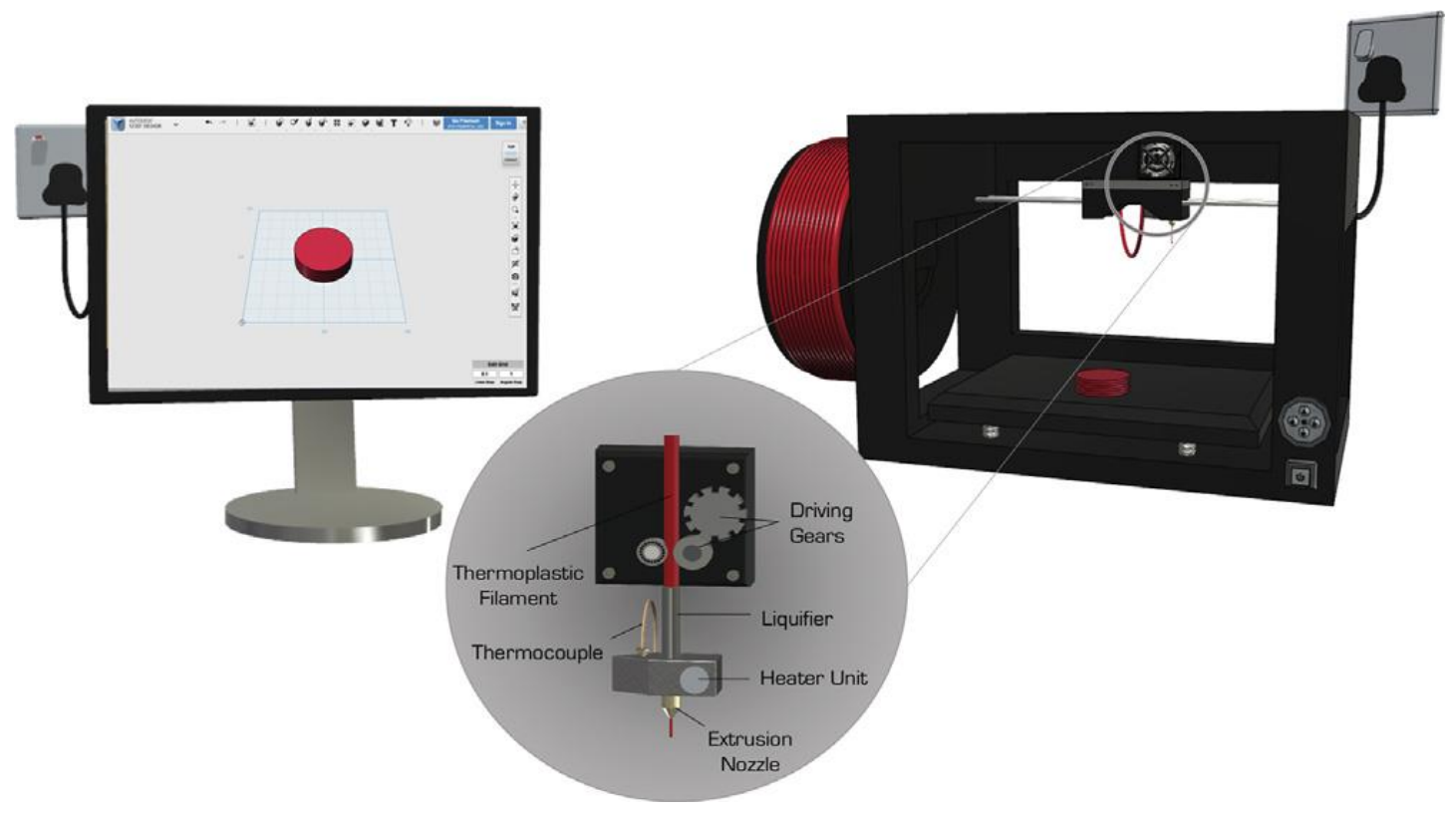

Figure 1. Graphical illustration of the FDM manner (Hospodiuk M. et al., 2017)

For remedy, surgeons discovered the opportunity of utilizing this generation for printing anatomical publications as well as organs in particularly tailored fashion for sufferers, allowing them to be educated for difficult surgical procedures. By integrating digital sensors, those fashions can offer quantitative evaluation at the surgical approach (e.g. length of a surgical operation and fulfillment quotes), and feature hence been termed "clever surgical aids". Additionally, organ fashions are founded totally on information of humans acquired with the use of properly-mounted imaging strategies, along with computed tomography (CT) and (MRI). Moreover, they can be applied for the method of checking out; a method known as bio-modelling. To elaborate, FDM 3D revealed induction port (IP) version, primarily based at the CT experiment of an affected person's trachea, can 
be discovered to be advanced into pharmacopoeia IPs, supplying greater perception into the in-vitro assessment of pressurized metered, dose inhalers.

However, in comparison with different 3DM technology, as an example, stereo-litho-graphy (SLA), is utilized for printing airway version for inspiratory float simulation or nasal casts for predicting drug deposition from nebulizers. For in-vitro assessments, FDM organs have been restrained. This may be accredited to the restricted decision of those printers, ensuing in a decrease in the precision of the printing or the want for using assist substances to create complicated fashions. Furthermore, modified osteoid casts were advanced as a shape of superior remedy for the use of FDM where these purposeful gadgets make use of low-depth pulsed ultrasound (LIPUS) for simulating bone recuperation. This, in turn, prompts mechanical pressure at the fractured bones' cells, lowering their restoration period by using as much as $38 \%$ (American Academy of Orthopaedic Surgeons, 2013). Moreover, FDM has been extensively used to layout affected person-unique scientific gadgets, which includes prosthetics and implants, in which the modifying of such gadgets is a demand (e.g. implants as well as transplants for plastic along with reconstructive surgical operation). An instance of such consists of the fabrication of functionalized ear prosthesis able to respond to temperature and stress. This excellent tool has the capacity to make use of mechanical strain and adjustments in temperature to result in electric ability, allowing it to reply to sound in sufferers who have loss of hearing. Additionally, FDM 3DM may be implemented for the engineering of bone tissue, wherein scaffolds have proven to be able to withstand excessive hundreds of mechanical pressures while keeping expanded tissue increase (Hajdu S. et al., 2015).

Previous issues recommendations on "Technical issues for scientific gadgets introduced synthetic", which set their expectancies for the usage of 3DM technology for the manufacture of scientific gadgets. Just a few of the published clinical implants of FDM (consisting of hip joints or cranial plates) were advertised, but not even one had been loaded.

\section{Plastic to Actives}

FDM-HME filaments created from thermoplastic substances are usually used as intermediaries in FDM printers. For instance, these thermoplastics include "polycarbonate (PC)", "polyvinyl alcohol (PVA)", "acrylonitrile butadiene styrene (ABS)" and "polylactic acid (PLA)". However, not even one of those industrial capillaries are accounted secure for human use. Thus, FDM substances are created in pharmaceutical studies thru a procedure referred to as warm soften thawing (HME). HME is extensively used inside the pharmaceutical enterprise to supply stable dispersants of secure polymers for the ingestion of humans to boom solubilities of weakly soluble capsules. The first step of the extrusion technique includes prepping the powder combination. The necessary portions of pills, excipients as well as polymers are cautiously measured followed by being blended (Wong K. Y. and Liu X., 2012). The homogenous aggregate is then lowered in the hopper, which moves in the extruder barrel. The powder gets exposed to an aggregate of excessive warmness and strain, main to its melting. In order to drive out the molten fabric from the metal nozzle, the rotary coil is used to shape the fabric into lengthy filaments of capillaries. Next, these are fed into an amassing tray, wherein they are left to chill. The capillaries are then sealed in suitable packaging and saved in a dryer to do away with any shape of dust.

The drug may be mixed in approaches: with the aid of integrating right into a powder aggregate previous to extrusion or by way of injecting extruded threads into the perfect drug answer, subjecting the drug to unfold severely within the filaments. A number of experiments have been attempted for impregnating tablets with the usage of passive diffusion. However, the principal drawback of this technique is the confined drug load $(<2 \%)$ related to it. HME can incorporate huge quantities of drugs, presenting extra flexibility for the dose. In addition, they may be tailored to generate preferred recipes appropriate for integrating diverse energetic elements, therefore averting the want to alternate the complete configuration. As such, capillaries may be used to mix special pills (eg. Prednisolone, theophylline, captopril and 5 aminosalicylic acids (5 ASA). For instance, a diameter of one. Seventy-five to three.00 \pm zero. $05 \mathrm{~mm}$ is needed to allow the weight of the cap inside the printhead. Stretch or decrease while heated, so selecting the precise nozzle length is essential. Furthermore, USA unification is essential to make certain a solid printing method due to the fact abnormalities can result in varying printing and reason failures in printing (Welling, et al., 2010).

This is by and large because of immoderate stress with the intention to save you the strings from bending well at the spool and prescribing its use. As such, this calls for a change of the polymer aggregate. In such instances, the addition of plasticizers allows to lessen the temperature of glass switch $(\mathrm{Tg})$ of the polymer and make the capillaries greater bendy.

However, the selection of the right kind and the quantity of plasticizers/sizes is important. This is especially due to the fact immoderate flexibility can reason the filament to emerge as very gentle and bend within the lobe 
and block it. As an end result, current paintings are aimed toward predicting thread printing via the usage of validation techniques to evaluate their mechanical houses.

\section{FDM Revolution and Its Transition}

Extensive research had been performed at the capacity of FDM to fabricate diverse styles of pharmaceutical merchandise including pills which is likewise called "printable" method pills, beads, catheters, defibrillators, topical mask, transcutaneous needles, vaginal earrings, intrauterine gadgets (IUDs), and subcutaneous gadgets (Boyer A. and Bonnin G., 2016). The essential incentives in the back of the most important use of FDM inside the pharmaceutical subject are summarized in Table 1.

Table 1. Parameters for evaluating the appropriateness of filaments to be used with FDM 3DM.

\begin{tabular}{|c|c|c|c|c|}
\hline Parameters & Risk if unmet & Validation methods & Required values & Proposed solutions \\
\hline Softness & Compression & $\begin{array}{l}\text { Driving gears in the } \\
\text { printing heads }\end{array}$ & $\begin{array}{l}\text { Tensile testing } \\
\text { Shore hardness }\end{array}$ & $\begin{array}{l}\text { Decreasing } \\
\text { plasticizer quantity }\end{array}$ \\
\hline Fragility & $\begin{array}{l}\text { Inappropriate loading in the } \\
\text { printing head. Breakage in the } \\
\text { liquefier while the printing or } \\
\text { loading processes occur }\end{array}$ & $\begin{array}{l}\text { Torsional strength Tensile } \\
\text { testing }\end{array}$ & $\begin{array}{l}\sim 0.15-0.2 \% \mathrm{~Pa} \\
\left(\begin{array}{lll}1 & 0 & 4\end{array}\right)\end{array}$ & $\begin{array}{l}\text { Utilization of } \\
\text { plasticizers }\end{array}$ \\
\hline & & Flexure testing & & \\
\hline $\begin{array}{l}\text { Stiffness } \\
\text { Prevents } \\
\text { filament } \\
\text { spooling }\end{array}$ & $\begin{array}{l}\text { Prevents the exiting of cloth from } \\
\text { the printing head }\end{array}$ & $\begin{array}{l}\text { Texture analysis } \\
\text { Dynamic mechanical } \\
\text { analysis (DMA) }\end{array}$ & $\sim 1000 \mathrm{~N} / \mathrm{m}$ Use & polymer blends \\
\hline $\begin{array}{l}\text { Diameter } \\
\text { consistency }\end{array}$ & $\begin{array}{l}\text { Alteration in feed Deformation in } \\
\text { prints Printing failure }\end{array}$ & $\begin{array}{l}\text { Digital caliper } \\
\text { Visual inspection }\end{array}$ & Constant diameter & Use of lubricant \\
\hline Diameter & $\begin{array}{l}\text { Failure to be loaded directly in } \\
\text { the printing head }\end{array}$ & $\begin{array}{l}\text { Ultrasonic thickness } \\
\text { Gauges. Digitalcalliper } \\
\text { Laser micrometer }\end{array}$ & $1.75-3.00 \mathrm{~mm}$ & $\begin{array}{l}\text { Selection of right } \\
\text { nozzle } \\
\text { (or die) size }\end{array}$ \\
\hline
\end{tabular}

\subsection{A Flexible Platform for Drug Shipping}

The range of human beings influences how pills paintings of their bodies. The human body is a complex system where variables such as genes, sickness/fitness and other aspects have a major role in drug conduct as well as drug pharmacodynamics. Therefore, unique people will want one of a kind doses structures or dosage paperwork, which aren't constantly effectively to be had. Studies have proven that maximum drug fulfilment fees are low at some stage in the early levels of medical trials. This results in a giant economic load for the medicinal enterprise.

After the creation of the Micro medicinal drug Initiative in 2017, there has been sizeable hobby inside the allocation of remedy (MinHwa Lee et al., 2016). Thus, pharmacists' purpose to create an unmarried platform that could tailor medicines to sufferers' wishes, choices and personality traits. Due to its specific functions and flexibility, FDM 3DM may be without difficulty tailored to fabricate truly any shape of the dose required with a selected model profile.

These traditional capsules are characterized by way of the appropriate spatial distribution of medication and sugars, which isn't always viable in maximum different production strategies. FDM generation has the capability to programmer drug transport structures to goal particular regions inside the human frame because of the extensive variety of polymeric matrices to be had. As such, FDM 3DM may be defined as a "dosage 
dispenser", permitting dosage bureaucracy to be synthetic at various doses, with launch profiles starting from instant to sustainable launch and changed without the want for added remedy, together with coating. Thus, this generation offers an extra state of the design technique for engineering pharmaceutical gadgets. In 2014, the primary try changed into made to analyze the 3DM FDM to put together oral prescribed drugs. Work has proven that print settings had been the important thing determinants of the drug model document. An instance is indoors layout or the proportion of leakage, which is the proportion of packaging within the outer envelope of 3-D published discs. Discs containing many less-clogging substances confirmed no quicker capsules, and people with better cloning confirmed better launch documents. This is specifically because of one of type swelling prices related to polymer density. Interestingly, at low dropout fees (much less than 20\%), the general density of 3-D published discs is decreased, accordingly improving their prosperity (Claire Morley et al., 2018).

Such a floating effect can be used for increasing the time of their stay in the stomach area, leading to prolonged drug release, a fact referred to as gastro protection. This discovery is especially important for weak essential drugs, as it enhances their bioavailability. However, this depends heavily on the patient's dietary consumption as well as the quantity of fluid in the abdomen that is being in the stomach, and therefore a significant variation in performance can be expected in the clinic. Instead, the devices routed to maximize the discharge of the drug were explored from the immediate release combinations (Figure 2A). With regulating the various features in the channels (such as configuration, height, and width), the drug version can be designed to satisfy the requirements and specifications. These multifaceted geometric patterns pose a hurdle to their products using traditional manufacturing procedures. Likewise, capsules with internal gaps, called the permissible gap, were fashioned to ease the fragmentation of fast release discs to avoid the necessity for disintegration (Figure 2B). By contrast, 3D model networks with controlled version characteristics vary (Ojakaa $\mathrm{D}$ et al., 2014). Table 2 summarizes the number one incentive at the back of the widespread use of FDM 3Dm for pharmaceutical studies, as shown below.

Table 2. The number one incentives at the back of the widespread use of FDM 3Dm for pharmaceutical studies.

\begin{tabular}{ll}
\hline Main motivations & Instances \\
\hline Drug synthesis & Remotely digitizing the plans for synthesizing and printing
\end{tabular}

Personalization of dosing flexibilities in drug loading
Customization of dosages bureaucracy for preclinical checking out in animals Patient-centric dosage bureaucracy Transforming pre-current dosage paperwork to

different kinds

Tuning the polymer matrix

Combining or greater capsules in unmarried dosage shapes of incompatible drugs

Subcutaneous devices, Intra-uterine devices, Topical masks Vaginal rings. Mouth guards.

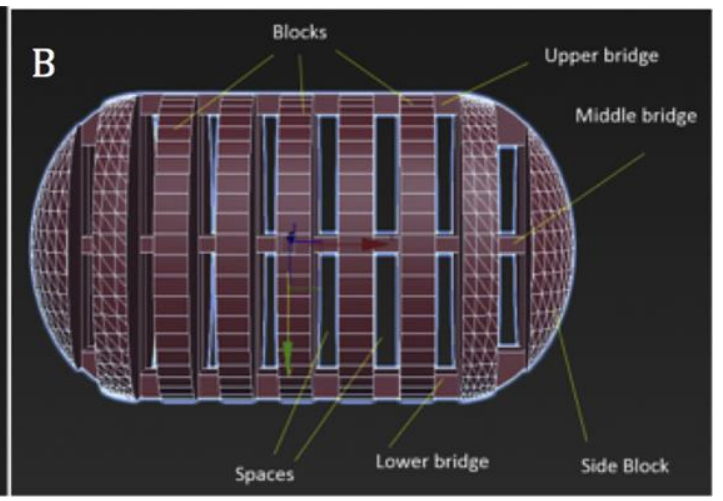

Figure 2. Diagram representing (A) channeled caplets and (B) gap lets prepared using FDM 3DM (Arafat Basel et al., 2018) 
Densities have been additionally fabricated. Mass, which may be managed by converting the dimensions of the 3D drugs, also can be applied to manipulate the drug launch. Results have proven that due to their better floor vicinity to mass ratio, smaller drugs showcase quicker drug launch. Likewise, the alternate inside the geometrical form of a 3D m pill turned into observed to steer the drug launch conduct (Tuma, J. M. and Pratt, J. M., 1982).

Five specific shapes, such as a dice, a torus, a sphere, a cylinder and a pyramid, have been discussed. The ratio of the floor location relative to the quantity of the pills can alter the time needed for the entire drug launch in in-vitro dissolution. Hence, while the slowest launch originates from the cylinder pill, the quickest drug launch changed into finding from the pyramid pill. Favorably, both, dose as well as weight accuracy is displayed by all 3DM pills. Realistically, despite the fact that regularly left out, the form, length, and shade of medicine may be great factors for the usefulness of a remedy proposal. An "open-label" test showed that patients tend to prefer ingesting a certain shaped pill over others. This thus influences their conformity to medicinal drugs. (Figure 3).

Results confirmed that the torus form became the maximum premier in phrases of ease of dealing with as well as ingesting. The shape which scored lowest in terms of the ingesting desire was the tilted diamond form. Due to the sharpness of the rims of this form, swallowing such tablets became problematic in perception by patients. Nevertheless, surprisingly, the sector pills had been more difficult to ingest in comparison to the diamond ones (Andreou E.P. and Philippou C.M., 2011).

In phrases of colours, most people of the sufferers believed that the addition of a colourant made the drugs extra attractive. This may be a predominant issue while managing positive age agencies, along with pediatric and geriatric sufferers, each of which usually be afflicted by terrible medicine acceptability. Unlike traditional manufacturing strategies, FDM 3DM permits the instantaneous variation of dosage bureaucracy to match any shape or trying out by using changing the 3D shape. As such, this may be taken into consideration as a main medical gain, hastening the drug improvement system with the aid of expediting its development into the pre-scientific and scientific levels. Whilst maximum studies on three-D prescribed drugs entail in vitro checking out, research the use of animal fashions were these days done.

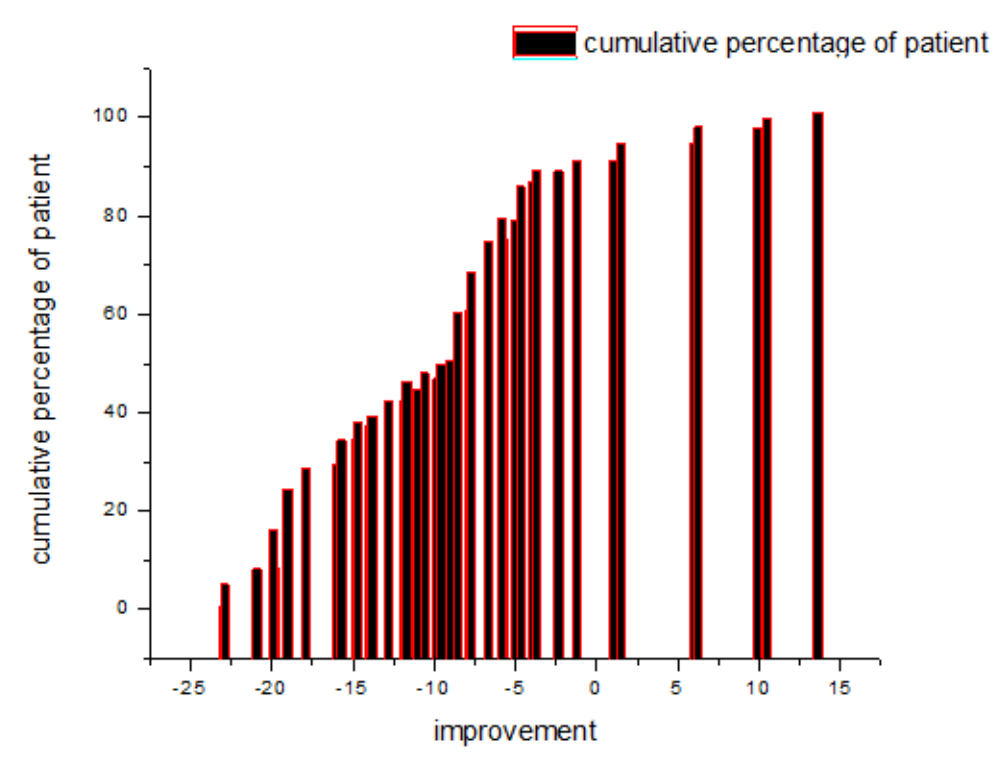

Figure 3. Patient reported outcome (PRO) scores showing the content for swallowing a tablet(s) (pre-swallowing) and the capability for swallowing the tablets (post-swallowing)

Due to the plainness of the FDM edition, 3D published discs can without problems be tailored to match a particular animal version. For instance, the scale of nine-hole drugs is in particular designed for management in mice. When examined, the consequences confirmed that every formulation confirmed a unique dissociation time, associated with polymer homes. Interestingly, tablets that remained intact confirmed no signs and symptoms of hint secretion or gastric emptying (Zhang B et al., 2016). This discovery could be very essential as it indicates that even though Volume nine drugs are frequently advertised as appropriate for rat control, their use is constrained most 
effective to instant launch programs. With past due or sustainable launch mixtures, the outcomes acquired the usage of the nine-length drugs can be deceptive due to the fact they can't empty the contagious location. They might also act as infectious formulations in mice rather. This discovery highlights particularly.

Common mistakes with the aid of formulation. Inappropriate choice of the suitable animal version for system checking out. As such, there's a want to assess opportunity dose paperwork or animal fashions for pre-scientific trying out. Unlike different 3DM strategies, FDM can put together pills containing a couple of polymers or energetic materials in an unmarried version, a manner called FDM. The feasibility of the use of FDM to put together managed shipping structures has been studied. Various preparations had been made (multilayer pills and Capo Duo). In multilayered caplets, every layer consists of one property, with the next layer made up of alternative. On the contrary, the Duo Caplets contains one drug in the middle of the tablet with a corona content (Al-Thyabat S et al., 2013).

While in vitro research confirmed that the discharge of the drug from multilayer pills changed into based totally completely on polymer homes, the outer layer changed into the step that confined the drug launch charge of Duo Caplets. FDM 3DM also can be used to create hole tablet caps with wonderful double cubicles. Individual elements may be synthetic the usage of the identical (Figure 4A) or special substances or thickness (Figure 4B), in which the discharge of the drug from every compartment may be released at distinctive time factors (Figure 4). Because those booths are sealed, they may be packed with powders or maybe drinks. This improvement is of super price for the established order of drug shipping structures that consist of numerous tablets (inclusive of metformin and glyburide) or drug doses, mainly given drugs may be shaped collectively despite the fact that they're no longer chemically well suited. The person compartments can be created utilizing the same (Fig. 4A) or distinctive (Fig. 4B) materials and/or thicknesses, whereby the sedate discharge from each compartment can be started at distinctive time points (Figure 5).

$\mathbf{A}$

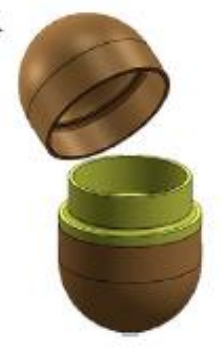

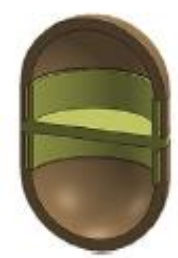

B

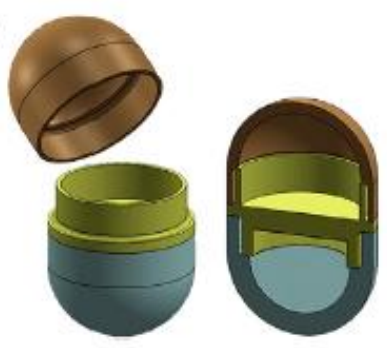

Figure 4. 3D revealed twin compartmental gadgets published the usage of the (A) equal or

(B) one-of-a-kind substances or thicknesses (Maroni A et al., 2017)

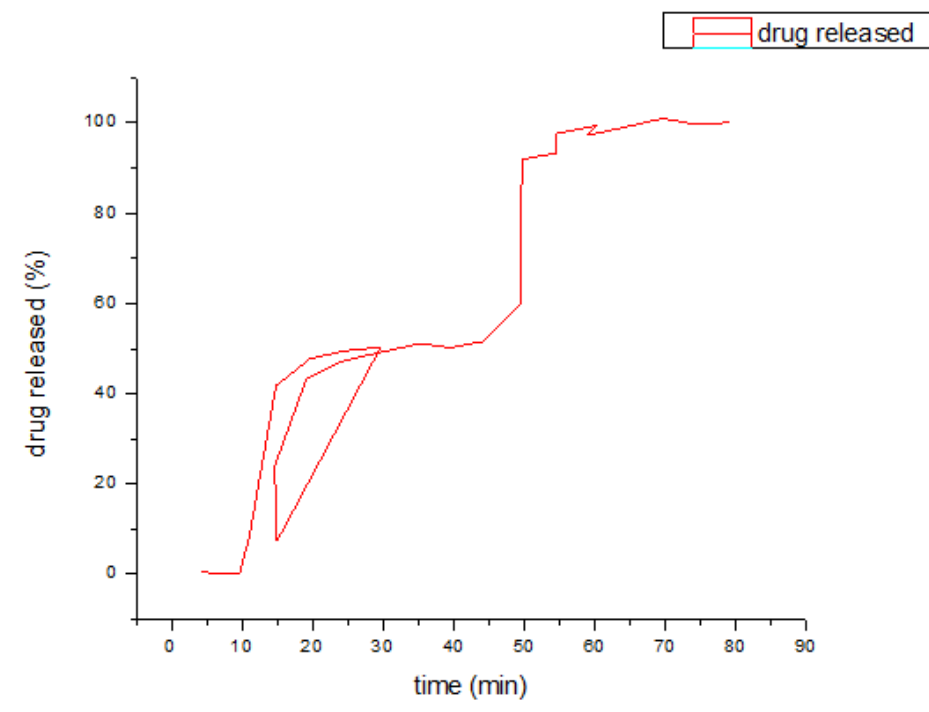

Figure 5. Dual compartmental devices' release profiles with varying wall thicknesses (i.e. 600 and $1200 \mu \mathrm{m}$ )

(Maroni A, 2017) 


\subsection{DM as a Digitized Tool}

Digital fitness is a multidisciplinary place linking fitness structures the usage of statistics acquired from fitness records (including ailment repute and genetic data) and verbal exchange technology (which include computational strategies and clever gadgets) to decorate remedy and offer custom designed styles of drug treatments. Plan desk, wearable or wearable sensors may be used to screen the fitness repute of the disorder remotely. The spinoff information can then be analyzed.

In 2017, FDA recommended an oral form containing an easily digestible sensor that digitally tracks whether a patient has administered the drug. The device works by sending a signal to the wearable patient patch which relates to the application of smartphones to enable the patient, profession or doctor to observe compliance. This idea can also be achieved using FDM, by following a 3D application of 3DM. While the design phase can be obvious for most applications, some patients may require more complex dosage or medical devices, requiring the use of 3D scanning. 3D scanning is a process involving the use of a 3D scanner to analyze and collect highly detailed data from an object (such as its shape, colour, and measurements) (Martins M., 2013).

For creating 3D numeric models, the collected data is then applied. Intrinsically, this can result in creating models for any realistic subject such as body parts. In the dental as well as audiology sectors, these applications have already been marketed, where the 3DM program is utilised to allocate hearing aids as well as invisible brackets. In pharmaceutical preparations, FDM can allow the manufacture of drug delivery materials adapted to patients (Figure 6). Its adjustment allows for improved hardware installation and provides long-lasting therapeutic effects. Furthermore, discomfort is less likely through these methods, which can then enhance the observance to the drug. 4DM is a new 3DM technology for manufacturing objects with conversion capability. In order to respond to a certain stimulus, these three-dimensional objects are programmed. Stimulus may include $\mathrm{pH}$, water, temperature and light, overall ones which cause the objects to experience predefined changes.

The programming procedure is based on two principles: Using smart materials and intelligent design of the 3D structure. 4D functional systems are manufactured with the combination of the basic concepts of FDM 3DM and polymer-responsive polymers. Poly (vinyl pyridine), a polymer that responds to $\mathrm{pH}$, was used as the backbone of the polymer in the current research. After printing, the three-dimensional printed structures were connected and intertwined, resulting in intelligent hydrogels where the later have demonstrated $\mathrm{pH}$-inverse responses. These are valuable for the flow regulation and totally porous membranes. This can definitely pave the way for further opportunities with the use of this technology even though this invention has not made use of a drug yet. This technology can assist in delivering local medicine to specific devices (Ahmadizar F et al., 2015). Therefore, enhanced techniques such as 4DM for development phases can offer enhanced as well as effective treatments, while decreasing the variations in therapeutic effects because of changes in patients. Overall, in clinics or hospitals, the complete phase-out will be allowed by the ease of handling as well as the compact sizes of FD, 3D printers. Enhancements to the health care systems with the adoption of this digital framework can then be possible while facilitating autonomy in patients during treatments as well as personalizing medicines and making them accessible to patients.

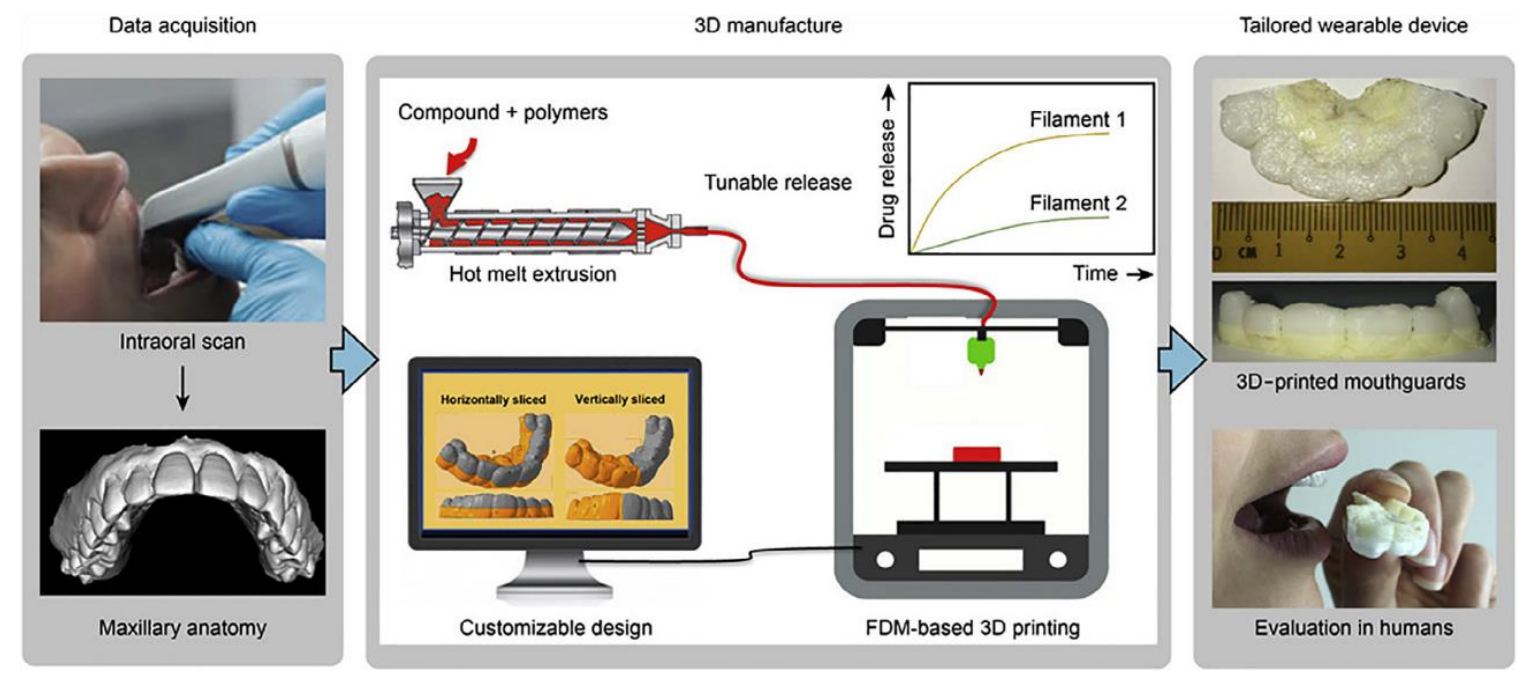

Figure 6. Flowchart representing the different phases of manufacturing wearable personalized oral delivery mouth guards with FDM 3DM (Liang Kun et al., 2018) 


\section{Problems as well as Obstacles}

Clearly, the combination of 3DM into scientific exercise can accelerate virtual fitness care revolution, converting the manner that pills and prescriptions are designed for sufferers. However, as with any new technology coming into the virtual fitness region, adoption is regularly gradual and may come alongside some of the demanding situations. The healthcare quarter has a terrible resistance to exchange, in all likelihood because of the reality that regulatory pointers and scientific approaches had been in the vicinity for decades. While that is comprehensible to hold affected person protection, it could frequently obstruct the equipped absorption of cutting-edge technological traits. In reality, with a view to boosting up the system of the usage of a 3DM era, you ought to offer a sturdy proof base to show that it'll be beneficial and secure no longer simplest for sufferers, however additionally for medical doctors who use generation each day (Towe S. L et al., 2015).

Hence, 3D published prescribed drugs have no longer been examined besides in laboratory or animal fashions. The handiest research concerning human topics had been an assessment of admission and an assessment observe. While each research covered the usage of FDM, this doesn't restrict using 3DM inside the virtual fitness of this AT, due to the fact different 3DM structures will also be beneficial. Moreover, at the identical time as the numerous researches have proven the plentiful benefits of FDM generation, none of those statistics had been included right into an unmarried look at to offer proof of medical advantages and private remedy effects. As such, up to now, the proposed beneficial capabilities of this method continue to be hypothetical, without a PK/ PD facts displaying the predicted absorption of advanced tablets and intestinal transit behaviours of those 3-dimensional published arrangements. However, with the fast development of this era and the modern strings made of GRASs commonly secure; it's miles best a depend on time till the 3-dimensional print arrangements input scientific trials.

First, should be in a position to triumph over the cutting-edge extraordinary issues and demanding situations and allow them to development closer to the "perfect three-D printer" phenomenon. First, considering the case that this approach includes a -step thermal system (extrusion and printing), each of which can be related to excessive temperatures (the maximum commonplace> $150^{\circ} \mathrm{C}$ ), there's an improved chance of degradation of the drug substance. By similarly trying out for brand new indicators that may lessen processing situations, FDM 3DM is probably so that you can print at room temperature inside the destiny. In addition to excessive temperatures, the HME step related to this method can take a long term, because the capillaries aren't effortlessly to be had and their printability is laid low with numerous functions.

Therefore, the FDM must undergo further progress. The requirements for pre-prepared threads must be discarded. As an alternative, printers must be designed for printing with the use of raw materials (such as powders or pellets). While the use of pellets as raw materials in some FDM printers is found, this technique is known to be in its early stages. In fact, complications such as slow material processing in common in the printers because of extended cooling periods as well as inadequate packaging of the layers in printing devices. In a positive, cost-effective manner, FDM 3D printers are suitable to be integrated into a pharmacy or hospital for customized dosages. The proposed course of treatment can include informing the healthcare practitioner with the patient-specific data for reviewing their medical history through digital or physical intervention. A digital prescription can then be produced by the doctor, which can be modified to the needs of the patient. This allows for a formula to be formulated and delivered to a locally based 3d FDM printer, which can dispense the medicine. Nevertheless, clear and concise compatible software platforms which allow interoperability amid varying techniques are required for achieving this. In addition, to protect patients' sensitive information and data, solutions to issues including data security as well as protection are needed. To do so, there is a requirement for communication between developers, technology innovators, health care practitioners and regulators to enable standardization in this sector.

At present, commercial 3D- FDM printers do not meet GMP specifications, which may make 3D printed products dangerous for humans. Consequently, these require the requirement for adequate quality control (QC) measures to warrant that regulatory strategies are met. First, isolating 3D printers can decrease the issue of product contamination as well as may offer enhanced command over changes in the ecological circumstances. Fortunately, because FDM 3D printed devices have excellent mechanical properties, tests such as rigidity as well as friability are unlikely. On another hand, while theoretically, 3-D must comprise of a "customized dose of medicine"; where validation must be made on the actual drug content. In addition, HME as well as FDM, both have shown a change in the physical nature of the drug molecules (such as crystalline/ non-crystalline conditions), thus affecting their stability, melting rates and solubility. Analytical testing is necessary for predicting drug performances (Zhao J et al., 2018). Nevertheless, methods of destructive characterization, for instance, high-performance liquid chromatography (HPLC), in vitro fertilization as well as 
dissociation testing, and ultraviolet spectroscopy, cannot be used in this scenario. As such, non-negative techniques, inclusive of analytical manner strategies (PAT), which include close to infrared spectroscopy.

In addition, converting print parameters (inclusive of form, length, or padding) may additionally exchange the general overall performance of your published three-D pill. Thus, this calls for the want for a predictive check of drug content material and bioavailability when it comes to the trade within the printing marketers. Best of all, the proposed analytical gadgets or techniques need to be included in the printers to make sure uniformity of the batch and expedite the very last dose distribution procedure.

\section{Conclusion}

3DM is about to revolutionize virtual healthcare. Due to its portability, simplicity and versatility, once can count on the FDM 3DM to be especially appropriate to be used inside the regions of virtual fitness. Unlike conventional production strategies, FDM 3DM allows the manufacturing of custom-designed, custom designed pharmaceutical merchandise with particular dosages, excessive manufacturing ability and sturdy mechanical residences. In addition, the manufacturing of complicated dosage paperwork may be accomplished, consisting of the absorption of many matrices and polymers, or with the aid of programming dosage paperwork to reveal precise consequences or focused on particular participants. The generation has developed similarly, making it viable to combine it with different technology for creating extra state-of-the-art and "clever" shipping structures. However, it's miles properly understood that virtual fitness isn't constrained to man or woman 3DM method, wherein different 3DM systems may show to be beneficial or may be advanced. However, all 3DM strategies are presently restrained by way of technical limitations and excellent manage, consequently proscribing their development. Once conquered, they may be moved ahead and carried out, converting the face of the medicinal enterprise and beginning a brand-new generation of virtual fitness.

\section{References}

Ahmadizar, F., Soltanian, K., Akhlaghiantab, F., \& Tsoulos, I. (2015). Artificial neural network development by means of a novel combination of grammatical evolution and genetic algorithm, Eng. Appl. Artif. Intell., 39, 1-13. https://doi.org/10.1016/j.engappai.2014.11.003

Ali, I., Ahmed, S., Tayyeh, H., Kolivand, H., \& Alkawaz, M. (2019). Virtual Human for Assisted Healthcare: Application and Technology. Encyclopedia of Computer Graphics and Games, Springer, Cham. https://doi.org/10.1007/978-3-319-08234-9_363-1

Al-Thyabat, S., T. Nakamura, E. S., \& Iizuka, A. (2013). Adaptation of minerals processing operations for lithium-ion (LiBs) and nickel metal hydride (NiMH) batteries recycling: Critical review. Minerals Engineering, Elsevier. 45, 4-17. https://doi.org/10.1016/j.mineng.2012.12.005

Andreou, E. P., \& Philippou, C. M. (2011). The Use and Effectiveness of Behavioral Modification Techniques in Achieving and Maintaining Normal Weight and Fitness - The Lifestyle Changes for Adults in Cyprus. Middlesex University.

Arafat, B., Magdalena, W., Abdullah, I., Robert, T. F., Mohammad, I., Waqar, A., ... Mohamed, A. A. (2018). Tablet fragmentation without a disintegrant: A novel design approach for accelerating disintegration and drug release from 3D printed cellulosic tablets. European Journal of Pharmaceutical Sciences, 118, 191-199. https://doi.org/10.1016/j.ejps.2018.03.019

Bhaduri, A. (2018). Mechanical Properties and Working of Metals and Alloys. Springer Series in Materials Science. https://doi.org/10.1007/978-981-10-7209-3

Boyer, A., \& Bonnin, G. (2016). Higher Education and the Revolution of Learning Analytics. International Council for open and Distace Education.

Claire, M., Maria, U., Jim, S., Gregory, M. P., \& Leigh, K. (2018). Emergency department crowding: A systematic review of causes, consequences and solutions. PLoS One, 13(8). https://doi.org/10.1371/journal.pone.0203316

Fina, F., Goyanes, A., Gaisford, S., \& Basit, A. W. (2012). Selective laser sintering (SLS) 3D printing of medicines. Int J Pharm, 285-293. https://doi.org/10.1016/j.ijpharm.2017.06.082

Hajdu, S. I., Vadmal, M., \& Tang, P. (2015). A note from history: Landmarks in history of cancer, part 7. Cancer, 121(15), 2480-2513. https://doi.org/10.1002/cncr.29365

Hospodiuk, M., Dey, M., Sosnoski, D., \& Ozbolat, I. T. (2017). The bioink: A comprehensive review on 
bioprintable materials. Biotechnology Advances, 35(2), 217-239.

https://doi.org/10.1016/j.biotechadv.2016.12.006

Kutz, M. ed. (2011). Applied plastics engineering handbook: processing and materials. William Andrew.

Kyobula, M., Aremu, A., Morgan, R. A., Ehab, S., Ricky, W., Ian, A., ... Clive, J. R. (2017). 3D inkjet printing of tablets exploiting bespoke complex geometries for controlled and tuneable drug release. J. Control Release, 261(10), 207-215. https://doi.org/10.1016/j.jconrel.2017.06.025

Liang, K., Simone, C., Davide, B., \& Jean-Christophe, L. (2018). 3D printing of a wearable personalized oral delivery device: A first-in-human study. Science advances, 4(5). https://doi.org/10.1126/sciadv.aat2544

Maroni, A., Melocchi, A., Parietti, F., Foppoli, A., Zema, L., \& Gazzaniga, A. (2017). 3D printed multi-compartment capsular devices for two-pulse oral drug delivery. Journal of Controlled Release, 268, 10-18. https://doi.org/10.1016/j.jconrel.2017.10.008

Martins M. (2013). A Simple Method for Assessment of MDR Bacteria for Over-Expressed Efflux Pumps. Open Microbiology, 22(7), 72-82. https://doi.org/10.2174/1874285801307010072

MinHwa, L., JinHyo, J. Y., OrcID, A. P., DongKyu, W., Fumio, K., Giovanni, S., ... Zhao, X. F. (2016). How to Respond to the Fourth Industrial Revolution, or the Second Information Technology Revolution? Dynamic New Combinations between Technology, Market, and Society through Open Innovation, MDPI. Journal of Open Innovation: Technol. Mark Complexity, 4(3). https://doi.org/10.3390/joitmc4030021

Mohamed, O. A., Masood, S. H., \& Bhowmik, J. L. (2015). Optimization of fused deposition modeling process parameters: a review of current research and future prospects. Advances in Manufacturing, 3, 42-53. https://doi.org/10.1007/s40436-014-0097-7

Ojakaa, D., Susan, O., \& Jordan, J. (2014). Factors affecting motivation and retention of primary health care workers in three disparate regions in Kenya. Hum Resource Health, 12-33. https://doi.org/10.1186/1478-4491-12-33

Towe, S. L., Hobkirk, A. L., Ye, D. G., \& Meade, C. S. (2015). Adaptation of the Monetary Choice Questionnaire to Accommodat Extreme Monetary Discounting in Cocaine Users. Psychol Addict Behav., 29(4), 1048-1055. https://doi.org/10.1037/adb0000101

Tuma, J. M., \& Pratt, J. M. (1982). Clinical child psychology practice and training: A survey. Clinical Child \& Adolescent Psychology, 137, 37-41.

Wagner, J. R., Mount, E. M., \& Giles, H. F. (2013). Extrusion: The Definitive Processing Guide and Handbook: Second Edition. Elsevier, Amsterdam.

Wink, M. (2015). Modes of Action of Herbal Medicines and Plant Secondary Metabolites. Medicines, 2(3), 251-286. https://doi.org/10.3390/medicines2030251

Wong, K. Y., \& Liu, X. (2012). Nanomedicine: A primer for surgeons. Pediatric Surgery International, 28(10), 943-951. https://doi.org/10.1007/s00383-012-3162-y

Zhang, B., Seong, B., Nguyen, V. D., \& Byun, D. (2016). 3D printing of high-resolution PLA-based structures by hybrid electrohydrodynamic and fused deposition modelling techniques. Journal Micromechanics Microengineering. https://doi.org/10.1088/0960-1317/26/2/025015

Zhao, J., Bo, R., Hou, Q., Cheng, M. M., \& Rosin, P. (2018). FLIC: Fast linear iterative clustering with active search. Comput. Vis. Media. Tsinghua University Press, 4(4), 333-348. https://doi.org/10.1007/s41095-018-0123-y

\section{Copyrights}

Copyright for this article is retained by the author(s), with first publication rights granted to the journal.

This is an open-access article distributed under the terms and conditions of the Creative Commons Attribution license (http://creativecommons.org/licenses/by/4.0/). 